\title{
Diagonalization of Hermitian Matrices
}

\section{By Carl-Erik Fröberg}

As is well known, real symmetric matrices can be diagonalized very efficiently by the method of Jacobi, consisting of repeated two-dimensional rotations. A similar method can be constructed for Hermitian matrices (cf. ref. 1, p. 160); in this case the corresponding transformation matrices are unitary.

Apart from trivial factors the general two-dimensional unitary matrix has the form

$$
U=\left(\begin{array}{cc}
\cos \varphi & -\sin \varphi \cdot e^{-i \theta} \\
\sin \varphi \cdot e^{i \theta} & \cos \varphi
\end{array}\right)
$$

A two-dimensional Hermitian matrix $H=\left(\begin{array}{cc}a & b-i c \\ b+i c & d\end{array}\right)$ is transformed to diagonal form by $U^{-1} H U=D$, where $\theta$ and $\varphi$ are defined by:

$$
\left\{\begin{aligned}
\operatorname{tg} \theta & =c / b \\
\operatorname{tg} 2 \varphi & =2 \sqrt{b^{2}+c^{2}} /(a-d)
\end{aligned}\right.
$$

The matrix elements of $D$ are:

$$
\left\{\begin{array}{l}
d_{11}=\frac{1}{2}(a+d+R) \\
d_{22}=\frac{1}{2}(a+d-R) \\
d_{12}=d_{21}=0
\end{array} \quad R^{2}=(a-d)^{2}+4\left(b^{2}+c^{2}\right)\right.
$$

This procedure is now applied to Hermitian matrices of arbitrary order. Let

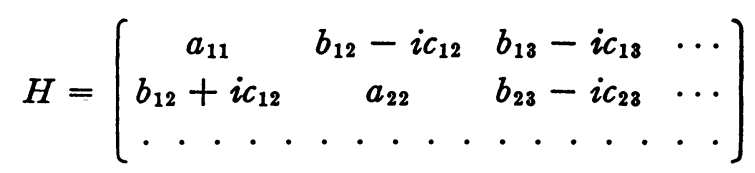

and define a real matrix $A=\left(\alpha_{i k}\right)$ in the following way:

$$
\left\{\begin{array}{l}
\alpha_{i k}=b_{i k} \quad i<k \\
\alpha_{i i}=a_{i i} \\
\alpha_{i k}=c_{k i} \quad i>k
\end{array}\right.
$$

After a two-dimensional rotation $(i, k)$ we arrive at a new Hermitian matrix $H^{\prime}$ and a corresponding real matrix $A^{\prime}=\left(\alpha^{\prime}{ }_{r s}\right)$. For computation of the elements $\alpha^{\prime}{ }_{r e}$ we introduce the following notations $(i<k)$ :

$$
\left\{\begin{array} { l } 
{ x _ { 1 } = \alpha _ { r i } \operatorname { c o s } \varphi } \\
{ x _ { 2 } = \alpha _ { i r } \operatorname { c o s } \varphi } \\
{ x _ { 3 } = \alpha _ { r k } \operatorname { c o s } \varphi } \\
{ x _ { 4 } = \alpha _ { k r } \operatorname { c o s } \varphi }
\end{array} \quad \left\{\begin{array} { l } 
{ y _ { 1 } = \alpha _ { r i } \operatorname { s i n } \varphi } \\
{ y _ { 2 } = \alpha _ { i r } \operatorname { s i n } \varphi } \\
{ y _ { 3 } = \alpha _ { r k } \operatorname { s i n } \varphi } \\
{ y _ { 4 } = \alpha _ { k r } \operatorname { s i n } \varphi }
\end{array} \quad \left\{\begin{array}{l}
z_{1}=y_{3} \cos \theta+y_{4} \sin \theta \\
z_{2}=-y_{3} \sin \theta+y_{4} \cos \theta \\
z_{3}=y_{1} \cos \theta-y_{2} \sin \theta \\
z_{4}=y_{1} \sin \theta+y_{2} \cos \theta
\end{array}\right.\right.\right.
$$

Received 10 December 1957. 
where

$$
\left\{\begin{aligned}
\operatorname{tg} \theta & =\alpha_{k i} / \alpha_{i k} \\
\operatorname{tg} 2 \varphi & =2 \sqrt{\alpha^{2}{ }_{i k}+\alpha^{2}{ }_{k i}} /\left(\alpha_{i i}-\alpha_{k k}\right)
\end{aligned}\right.
$$

Then we have for $r<i$ or $r>k$ : for $i<r<k$ :

$$
\left\{\begin{array} { l } 
{ \alpha ^ { \prime } { } _ { r i } = x _ { 1 } + z _ { 1 } } \\
{ \alpha ^ { \prime } { } _ { i r } = x _ { 2 } + z _ { 2 } } \\
{ \alpha ^ { \prime } { } _ { r k } = x _ { 3 } - z _ { 3 } } \\
{ \alpha ^ { \prime } { } _ { k r } = x _ { 4 } - z _ { 4 } }
\end{array} \quad \left\{\begin{array}{l}
\alpha_{r i}^{\prime}=x_{1}-z_{2} \\
\alpha^{\prime}{ }_{i r}=x_{2}+z_{1} \\
\alpha_{r k}^{\prime}=x_{3}-z_{4} \\
\alpha^{\prime}{ }_{k r}=x_{4}+z_{3}
\end{array}\right.\right.
$$

and further $\alpha^{\prime}{ }_{i i}=\frac{1}{2}\left(\alpha_{i i}+\alpha_{k k}+R\right), \alpha^{\prime}{ }_{k k}=\frac{1}{2}\left(\alpha_{i i}+\alpha_{k k}-R\right), \alpha^{\prime}{ }_{i k}=\alpha^{\prime}{ }_{k i}=0$. Here $R^{2}=\left(\alpha_{i i}-\alpha_{k k}\right)^{2}+4\left(\alpha^{2}{ }_{i k}+\alpha^{2}{ }_{k i}\right)$. When both $r$ and $s$ differ from $i$ and $k$ we have $\alpha_{r s}^{\prime}=\alpha_{r s}$. The indices $i$ and $k$ can be chosen conveniently, e.g. to fulfill the condition $\left|\alpha_{i k}\right|+\left|\alpha_{k i}\right|=\max$.

The whole procedure is now repeated until all eigenvalues have been established with sufficient accuracy in the main diagonal.

This method has been successfully tested on SMIL, the electronic computer of Lund University, up to $n=15$. The matrices had all elements in the main diagonal $=1$, all upper-diagonal elements $=1-i$ and all lower-diagonal elements $=1+i$. The eigenvalues of these matrices can also be computed directly: $\lambda_{k}(n)=\cot (\pi(4 k+1) / 4 n) ; k=0,1, \cdots(n-1)$. An accuracy of about 8 decimal digits was easily obtained; further it turned out that the time consumed was about 4 times longer than for the corresponding diagonalization of real symmetric matrices.

Department of Numerical Analysis

Sölvegatan 14

Lund, Sweden

1. A. S. Householder, Principles of Numerical Analysis, McGraw-Hill Book Co., Inc., New York, 1953.

2. E. G. Kogbetliantz, "Solution of linear equations by diagonalization of coefficients matrix," Quart. Appl. Math., v. 13,1955, p. 123-132. 47-52.

3. J. GreenstadT, "A method for finding roots of arbitrary matrices," $M T A C$, v. 9, 1955,

4. M. Lotkın, "Characteristic values of arbitrary matrices," Quart. Appl. Math., v. 14, 1956, p. $267-275$.

\section{REVIEWS AND DESCRIPTIONS OF TABLES AND BOOKS}

86[C].-NBS Applied Mathematics Series, No. 53, Table of Natural Logarithms for Arguments Between Five and Ten to Sixteen Decimal Places, U. S. Government Printing Office, Washington, D. C., 1958, xiii +506 p., $26 \mathrm{~cm}$. Price $\$ 4.00$.

This book tabulates $\log _{e} x, x=5(.0001) 10,16 \mathrm{D}, \log _{e} x, x=2(1) 10,40 \mathrm{D}$, and $\log _{e}(1+x)$ and $-\log _{e}(1-x), x=10^{-n}\left(10^{-n}\right) 10^{-n+1}, n=1(1) 13,25 \mathrm{D}$. In addition there are sixteen constants given to $20 \mathrm{D}$.

With AMS 31 (see $M T A C$, v. 8 , Rev. 1167, 1954, p. 76) these give $\log _{e} x$ for $x=0(.0001) 10,16 \mathrm{D}$ and enough auxiliary material to make the computation of any natural logarithm to accuracy compatible with the tables reasonably con- 